01

\title{
Спектры комбинационного рассеяния азота, диоксида углерода и водорода в окружении метана
}

\author{
(С) Д.В. Петров ${ }^{1,2}$, И.И. Матросов ${ }^{1}$, Д.О. Сединкин ${ }^{1}$, А.Р. Зарипов ${ }^{1}$ \\ ${ }^{1}$ Институт мониторинга климатических и экологических систем Сибирского отделения РАН, \\ 634055 Томск, Россия \\ ${ }^{2}$ Национальный исследовательский Томский государственный университет, \\ 634050 Томск, Россия \\ e-mail: Dpetrov@imces.ru
}

Поступила в редакцию 05.07.2017 г.

Исследованы изменения спектров комбинационного рассеяния (КР) $\mathrm{N}_{2}, \mathrm{H}_{2}$ и $\mathrm{CO}_{2}$ в диапазоне 200-3800 $\mathrm{cm}^{-1}$ в зависимости от концентрации окружающих молекул $\mathrm{CH}_{4}$ при фиксированном давлении среды $25 \mathrm{~atm}$ и температуре $300 \mathrm{~K}$. Установлено, что в среде $\mathrm{CH}_{4}$ спектральные характеристики чисто вращательных линий $\mathrm{H}_{2}$ изменяются пренебрежимо мало, в то время как $Q$-ветви ферми-дублета $\mathrm{CO}_{2}$ $v_{1} / 2 v_{2}$ сужаются, а частотные сдвиги его высокочастотного компонента и полосы $v_{1} \mathrm{~N}_{2}$ уменьшаются. Помимо этого, в данных условиях отношение интенсивностей $Q$-ветви ферми-дублета $\mathrm{CO}_{2}$ изменяется пропорционально концентрации окружающих молекул $\mathrm{CH}_{4}$. Полученные данные будут востребованы при диагностике состава природного газа с помощью спектроскопии КР.

DOI: $10.21883 / O S .2018 .01 .45350 .158-17$

\section{Введение}

Природный газ (ПГ) представляет собой многокомпонентную газовую смесь, широко используемую в качестве экологически чистого топлива и сырья для химической промышленности. Помимо $\mathrm{CH}_{4}$, доля которого может достигать $\sim 95 \%$, в состав ПГ входят его гомологи $\left(\mathrm{C}_{2} \mathrm{H}_{6}, \mathrm{C}_{3} \mathrm{H}_{8}, \mathrm{C}_{4} \mathrm{H}_{10}, \mathrm{C}_{5} \mathrm{H}_{12}\right.$ и т.д.), а также неуглеводородные соединения (как правило, $\mathrm{N}_{2}, \mathrm{CO}_{2}$, $\mathrm{H}_{2}$ ). Содержание этих компонентов в ПГ неодинаково для различных месторождений, поэтому его энергетическая и химическая ценность может сильно различаться. В связи с этим определение компонентного состава ПГ имеет большое значение как для покупателей газа, так и для его поставщика.

На сегодняшний день активно развивается метод диагностики состава ПГ, основанный на спектроскопии комбинационного рассеяния света (КР) [1-8]. Данный метод обладает такими преимуществами, как возможность проведения экпресс-анализа, бесконтактность, отсутствие расходных материалов, а также одновременный контроль всех молекулярных компонентов среды, концентрация которых превышает порог чувствительности аппаратуры.

Несмотря на относительно низкий уровень информативных сигналов, на сегодняшний день экспериментально подтвержденная предельная чувствительность метода КР-газоанализа при диагностике ПГ составляет $0.002 \%$ [1]. В свою очередь, для повышения точности данного метода необходимы данные, касающиеся изменения спектров КР основных компонентов ПГ в зависимости от условий, в которых они находятся непосредственно в анализируемой пробе ПГ. Ранее нами было установлено, что спектр КР основного компонента ПГ - метана, включая полосы $\left(v_{1}, v_{2}, v_{3}\right.$,
$2 v_{2}$ и $\left.2 v_{4}\right)$, имеет достаточно сильную зависимость от температуры [9] и давления [10]. На основании этого было сделано заключение, что для повышения достоверности КР-газоанализ ПГ необходимо осуществлять при фиксированных значениях данных параметров. Поскольку в магистральных трубопроводах ПГ находится под давлением не менее $25 \mathrm{~atm}$, то определение его состава с помощью КР-газоанализатора пробоотборного типа целесообразно проводить при давлении $25 \mathrm{~atm}$ для гарантированного обеспечения идентичных условий, в которых находятся компоненты ПГ. В свою очередь оптимальной температурой с точки зрения технической реализации является $\sim 300 \mathrm{~K}$.

Следующим шагом в данном направлении является определение влияния $\mathrm{CH}_{4}$ (как доминирующего компонента ПГ) на спектры КР других компонентов ПГ в обозначенных выше условиях. Несмотря на то, что в литературе представлены данные, касающиеся влияния $\mathrm{CH}_{4}$ на фундаментальные полосы $\mathrm{N}_{2}[11,12], \mathrm{CO}_{2}$ [13] и $\mathrm{C}_{2} \mathrm{H}_{6}$ [14] при давлениях до $3000 \mathrm{~atm}$, требуемая информация из них получена быть не может, ввиду больших разбросов полученных значений при малых давлениях $(<25 \mathrm{~atm})$.

В этой связи данная работа посвящена исследованию влияния $\mathrm{CH}_{4}$ на спектры КР основных неуглеводородных компонентов ПГ $\left(\mathrm{N}_{2}, \mathrm{CO}_{2}\right.$ и $\left.\mathrm{H}_{2}\right)$ при давлении $25 \mathrm{~atm}$ и температуре $300 \mathrm{~K}$.

\section{Экспериментальная часть}

Исследования были выполнены с помощью экспериментальной установки на основе разработанного КР-спектрометра, ранее использовавшегося в работе [1]. Коллимированное возбуждающее излучение диаметром 
$\sim 3 \mathrm{~mm}$, генерируемое твердотельным лазером с диодной накачкой (SDL-532-2000) с выходной мощностью $2 \mathrm{~W}$ на длине волны $532 \mathrm{~nm}$ направлялось внутрь газовой кюветы объемом $\sim 10 \mathrm{~cm}^{3}$. Рассеянное излучение собиралось в направлении, перпендикулярном направлению распространения лазерного излучения с помощью пары идентичных линзовых объективов с относительным отверстием $f / 1.8$ и фокусным расстоянием $50 \mathrm{~mm}$. Для разложения собранного излучения в спектр использовался специализированный спектральный прибор „МКР-2“ с входным относительным отверстием $f / 1.8$. Регистрация спектров проводилась с помощью ПЗС-матрицы Hamamatsu S10141 $(2048 \times 256$ ріх $)$ с охлаждением Пельтье до $-10^{\circ} \mathrm{C}$, работающей в режиме ПЗС-линейки за счет вертикального биннинга зарядов. С данной матрицей при использовании входной щели шириной $40 \mu \mathrm{m}$ и дифракционной решетки 1600 grooves $/ \mathrm{mm}$ обеспечивалось разрешение $\sim 6 \mathrm{~cm}^{-1}$ при дисперсии $1.8 \mathrm{~cm}^{-1} / \mathrm{pix}$ и одновременной регистрации спектрального диапазона 200-3800 $\mathrm{cm}^{-1}$. Частотная калибровка спектрометра была проведена по спектру излучения неоновой лампы [10].

На данном оборудовании были зарегистрированы спектры КР $\mathrm{N}_{2}, \mathrm{CO}_{2}$ и $\mathrm{H}_{2}$, а также их смесей с различной концентрацией $\mathrm{CH}_{4}(20,40,60,80$ и 96\%). Требуемое давление $(25 \mathrm{~atm})$ исследуемых газовых сред контролировалось манометром с погрешностью < $0.02 \mathrm{~atm}$. Чистота используемых газов > 99.9\%. Время экспозиции каждого спектра составляло $300 \mathrm{~s}$, при этом газовая кювета была термостабилизирована на $300 \pm 1 \mathrm{~K}$.

\section{Результаты и обсуждение}

На рис. 1 представлены нормированные спектры КР $\mathrm{N}_{2}, \mathrm{CO}_{2}$ и $\mathrm{H}_{2}$. В исследуемом диапазоне частотных сдвигов $\mathrm{N}_{2}$ имеет только одну фундаментальную колебательно-вращательную полосу $v_{1}$ с неразрешенной $Q$-ветвью, максимум которой наблюдался на $2328.72 \mathrm{~cm}^{-1}$. Спектр $\mathrm{CO}_{2}$ в данном диапазоне представляет собой ферми-дублет, являющийся результатом

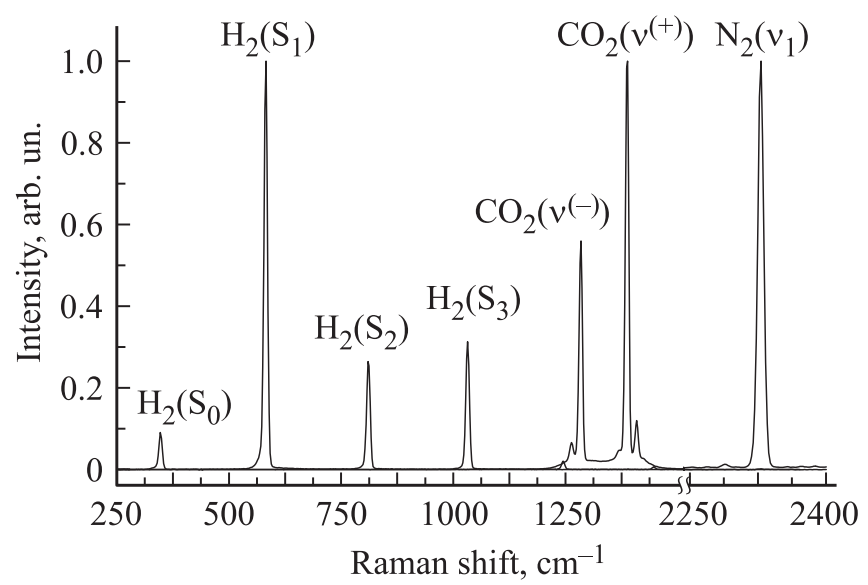

Pис. 1. Спектры КР $\mathrm{N}_{2}, \mathrm{H}_{2}, \mathrm{CO}_{2}$ в спектральном диапазоне $250-2400 \mathrm{~cm}^{-1}$.
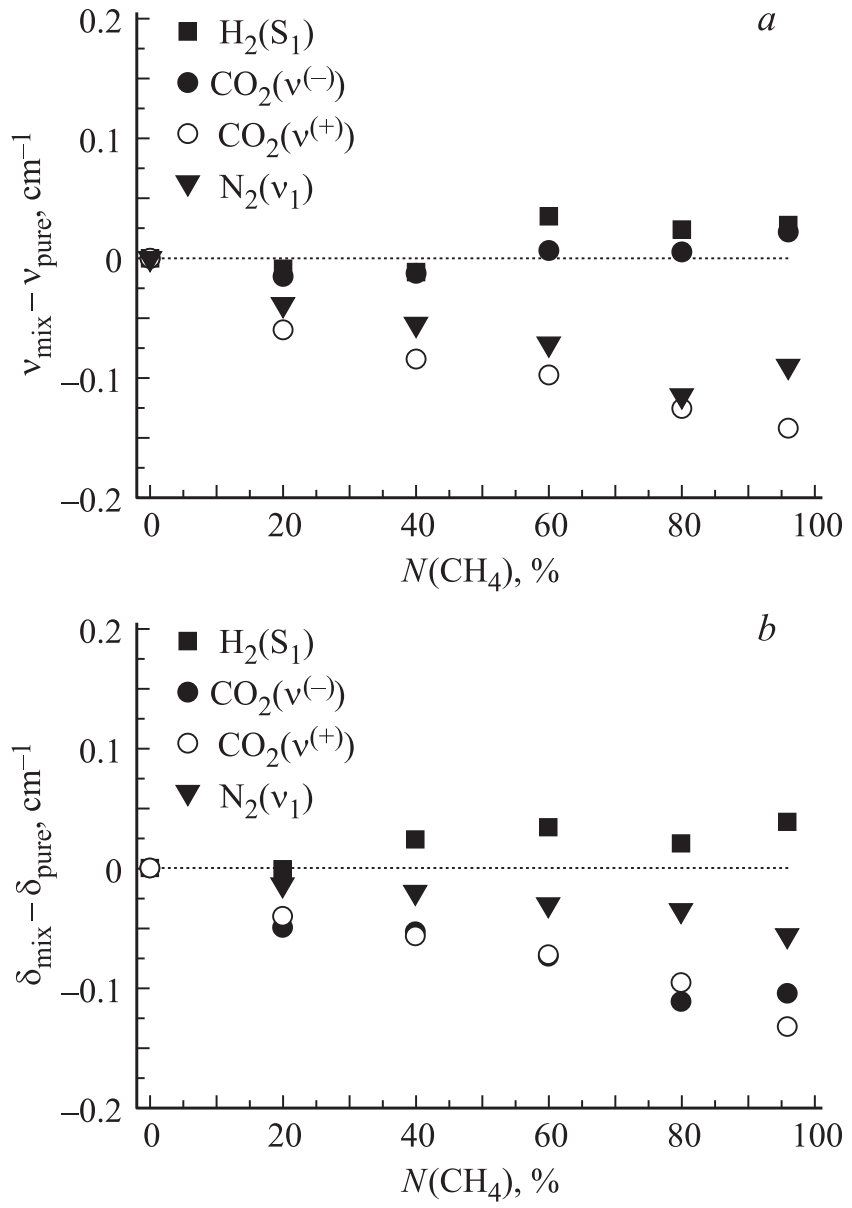

Рис. 2. Изменение частотных сдвигов $(a)$ и полуширин $(b)$ $Q$-ветвей $\mathrm{N}_{2}$ и $\mathrm{CO}_{2}$, а также чисто вращательных линий $\mathrm{H}_{2}$ в зависимости от концентрации окружающих молекул $\mathrm{CH}_{4}$.

взаимодействия полосы $v_{1}$ с обертоном $2 v_{2} \quad[15,16]$. Поскольку в данном случае нельзя однозначно связать наблюдаемые $Q$-ветви с соответствующими колебаниями, далее мы будем упоминать их как низкочастотный $v^{(-)}\left(1284.72 \mathrm{~cm}^{-1}\right)$ и высокочастотный $v^{(+)}\left(1388.01 \mathrm{~cm}^{-1}\right)$ компоненты ферми-дублета $v_{1} / 2 v_{2}$. В свою очередь, спектр КР $\mathrm{H}_{2}$ представлен в данном диапазоне только чисто вращательными линиями: $S_{0}\left(354.82 \mathrm{~cm}^{-1}\right), S_{1}\left(587.40 \mathrm{~cm}^{-1}\right), S_{2}\left(815.01 \mathrm{~cm}^{-1}\right)$ и $S_{3}\left(1034.86 \mathrm{~cm}^{-1}\right)$. Отметим, что процедура вычисления компонентного состава ПГ осуществляется в диапазоне $200-2600 \mathrm{~cm}^{-1}[17]$, и в этой связи колебательно-вращательная полоса водорода $v_{1}$, имеющая частотный сдвиг $4156 \mathrm{~cm}^{-1}$, не представляет практического интереса в данном контексте.

В ходе работы были исследованы частотные сдвиги и полуширины (ширины на полувысоте) обозначенных выше трех $Q$-ветвей $\left(\mathrm{N}_{2}\left(v_{1}\right), \mathrm{CO}_{2}\left(v^{(-)}\right), \mathrm{CO}_{2}\left(v^{(+)}\right)\right)$и четырех чисто вращательных линий $\mathrm{H}_{2}$ в зависимости от концентрации окружающих молекул $\mathrm{CH}_{4}$. Для повышения точности была использована их аппроксимация гауссовым контуром. Среднеквадратическое отклонение в данном случае не превышало $\sim 0.06 \mathrm{~cm}^{-1}$. В ходе 


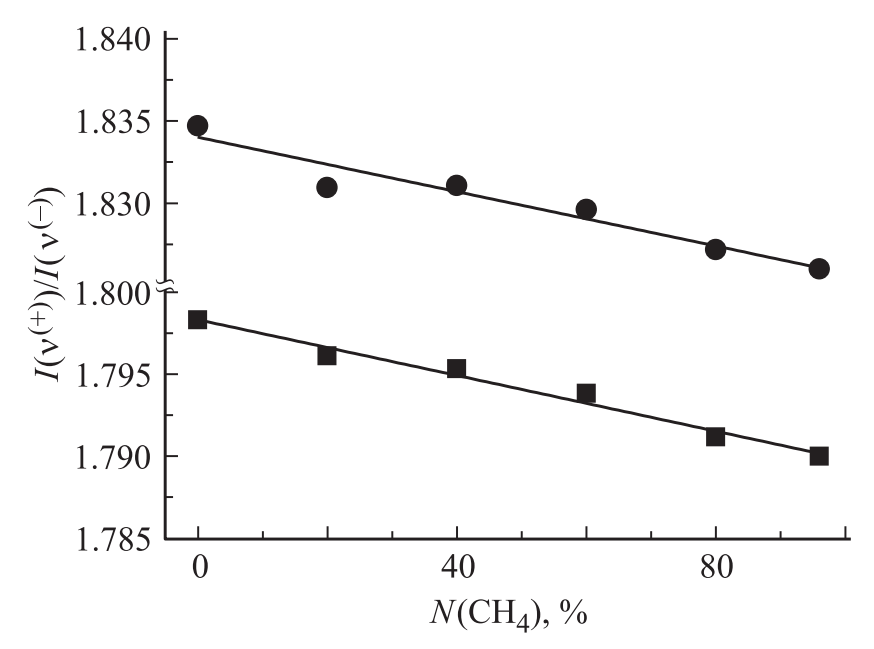

Рис. 3. Отношение интегральных (квадраты) и пиковых (кружки) интенсивностей $Q$-ветвей ферми-дублета $\mathrm{CO}_{2}$ в зависимости от концентрации окружающих молекул $\mathrm{CH}_{4}$.

исследования было установлено, что $\mathrm{CH}_{4}$ одинаково влияет на все линии $\mathrm{H}_{2}$. В этой связи в полученных результатах (рис. 2) приведены данные только для наиболее интенсивной линии $S_{1}\left(587.40 \mathrm{~cm}^{-1}\right)$. Также необходимо отметить, что перед анализом спектров $\mathrm{CO}_{2}$ в смеси с $\mathrm{CH}_{4}$ проводилась процедура вычитания из них спектра чистого $\mathrm{CH}_{4}$ ввиду перекрытия полос $\mathrm{CO}_{2} \mathrm{c}$ крылом полосы $v_{2} \mathrm{CH}_{4}\left(1536 \mathrm{~cm}^{-1}\right)$.

Согласно полученным данным при увеличении концентрации $\mathrm{CH}_{4}$ от 0 до $96 \%$ положение $Q$-ветви $\mathrm{N}_{2}\left(v_{1}\right)$ смещается в область меньших частотных сдвигов на $\sim 0.10 \mathrm{~cm}^{-1}$. В аналогичных условиях $Q$-ветви ферми-дублета $\mathrm{CO}_{2}$ ведут себя по-разному: частотный сдвиг высокочастотного компонента $v^{(+)}$уменьшается на $\sim 0.15 \mathrm{~cm}^{-1}$, а положение низкочастотного компонента $v^{(-)}$практически не изменяется (в пределах погрешности измерений). В свою очередь влияние окружения $\mathrm{CH}_{4}$ в данных условиях на положения вращательных линий $\mathrm{H}_{2}$ было пренебрежимо мало.

Касательно изменения полуширин анализируемых линий и $Q$-ветвей можно отметить следующее. Наиболее заметное влияние $\mathrm{CH}_{4}$ оказывает на $Q$-ветви $\mathrm{CO}_{2}$, что отражается в их сужении на $\sim 0.1 \mathrm{~cm}^{-1}$. При этом необходимо отметить, что в отличие от изменения частотных сдвигов, изменение их полуширин идентичное. $Q$-ветвь $\mathrm{N}_{2}$ в данных условиях также подвержена сужению, однако на меньшую величину $\left(\sim 0.06 \mathrm{~cm}^{-1}\right)$. В свою очередь, чисто вращательные линии $\mathrm{H}_{2}$ при увеличении концентрации $\mathrm{CH}_{4}$ испытывали уширения на $\sim 0.04 \mathrm{~cm}^{-1}$. Необходимо отметить, что несмотря на то, что данные для $\mathrm{N}_{2}$ и $\mathrm{H}_{2}$ находятся в пределах погрешности измерений, все наблюдаемые тренды соответствуют изменению плотности среды. Так, при увеличении доли $\mathrm{CH}_{4}$ в смеси с $\mathrm{H}_{2}$ среда становится более плотной, в результате чего линии $\mathrm{H}_{2}$ уширяются, а в случаях с $\mathrm{CO}_{2}$ и $\mathrm{N}_{2}-$ менее плотной, что приводит к сужению их $Q$-ветвей. При этом, поскольку плотность $\mathrm{CO}_{2}$ по сравнению с $\mathrm{N}_{2}$ больше отличается от плотности $\mathrm{CH}_{4}$, то и наблюдаемое изменение полуширин $Q$-ветвей $\mathrm{CO}_{2}$ больше. Данная версия также находит свое подтверждение в работах $[11,13,18]$.

В ходе работы также были исследованы эффекты перераспределения интенсивностей между $Q$-ветвями фермидублета $\mathrm{CO}_{2}$ и отдельными вращательными линиями $\mathrm{H}_{2}$ в случае нахождения данных молекул в среде с различным содержанием $\mathrm{CH}_{4}$. Для этого из всех полученных
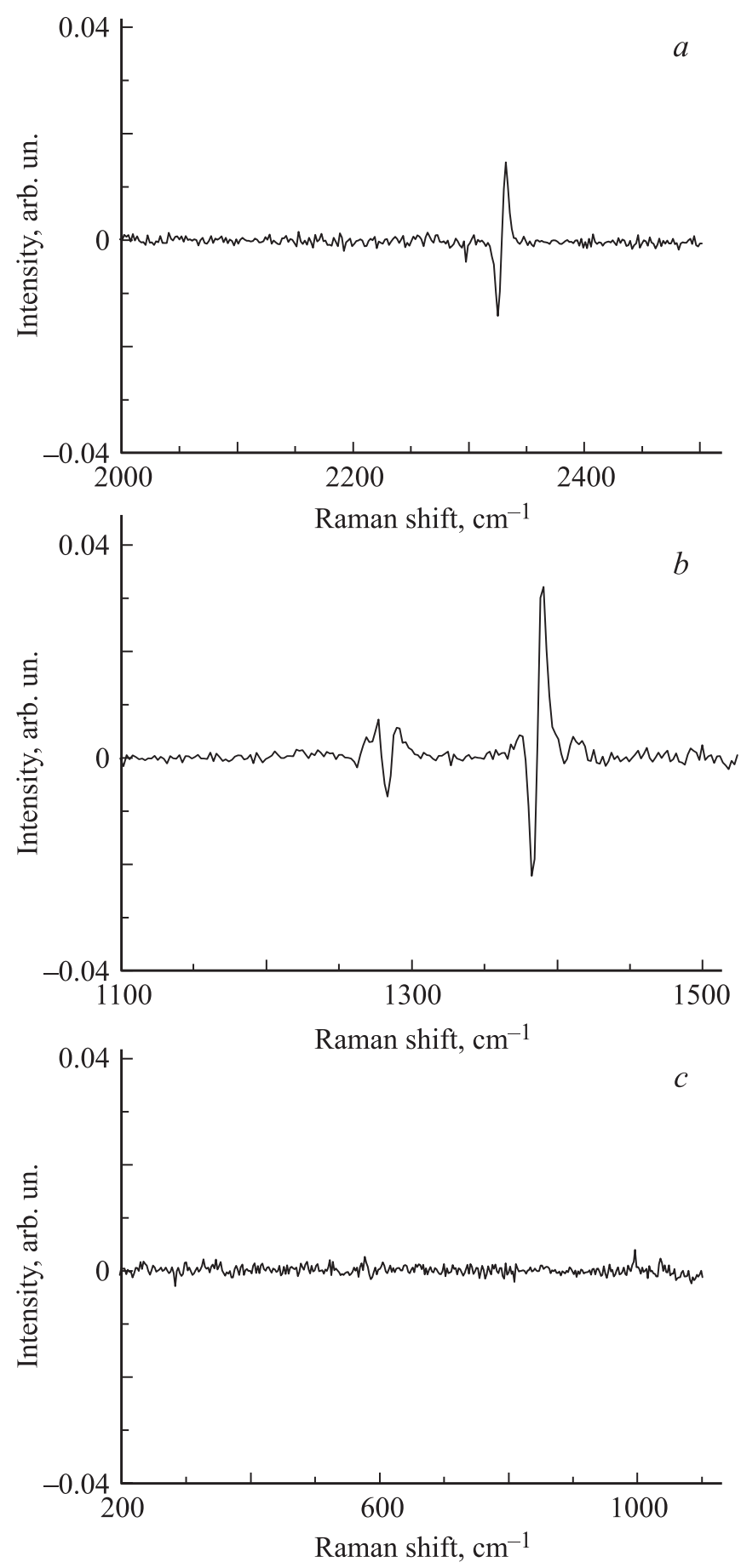

Рис. 4. Разность между спектрами КР $\mathrm{N}_{2}(a), \mathrm{CO}_{2}(b), \mathrm{H}_{2}(c)$, полученными для чистых газов и в смеси с $\mathrm{CH}_{4}(96 \%)$. 
спектров КР $\mathrm{CO}_{2}$ определялось отношение интенсивностей $I\left(v^{(+)}\right) / I\left(v^{(-)}\right)$, а из спектров КР $\mathrm{H}_{2}$ определялись отношения $I\left(S_{1}\right) / I\left(S_{0}\right), I\left(S_{1}\right) / I\left(S_{2}\right), I\left(S_{1}\right) / I\left(S_{3}\right)$. Согласно полученным данным, перераспределения интенсивностей чисто вращательных линий $\mathrm{H}_{2}$ ввиду различного окружения не наблюдалось. В свою очередь, для $Q$-ветвей ферми-дублета $\mathrm{CO}_{2}$ это имело место (рис. 3), причем как для пиковых интенсивностей, так и для интегральных. Отметим, что для $v^{(-)}$интегрирование интенсивностей проводилось в диапазоне $1275-1295 \mathrm{~cm}^{-1}$, для $v^{(-)}-$в диапазоне $1377-1397 \mathrm{~cm}^{-1}$. В свою очередь, пиковые интенсивности определялись из гауссовых контуров, аппроксимирующих данные $Q$-ветви. Из полученных данных, представленных на рис. 3, видно, что с увеличением концентрации $\mathrm{CH}_{4}$ отношение $I\left(v^{(+)}\right) / I\left(v^{(-)}\right)$уменьшается. Поскольку выполненные измерения являются относительными, то из экспериментальных данных нельзя однозначно сказать, что указанный эффект вызван либо увеличением интенсивности низкочастотного компонента $v^{(-)}$, либо уменьшением интенсивности высокочастотного компонента $v^{(+)}$. Однако согласно механизму взаимодействия полос $v_{1}$ и $2 v_{2}$, описанному в [15], наблюдаемое уменьшение расстояния между $Q$-ветвями дублета при уменьшении плотности среды (за счет уменьшения концентрации $\mathrm{CO}_{2}$ в смеси) свидетельствует об ослаблении ферми-резонанса между данными полосами. Отметим, что аналогичные эффекты мы ранее наблюдали $[10,19]$ при исследовании спектров $\mathrm{KP} \mathrm{CH}_{4}$ и $\mathrm{CO}_{2}$ в зависимости от давления.

Для наглядного подтверждения изменения спектров КР анализируемых молекул в окружении $\mathrm{CH}_{4}$ была получена разность между нормированными спектрами с концентрацией $\mathrm{CH}_{4} \mathrm{O}$ и $96 \%$. Из представленных на рис. 4 данных видно, что несмотря на то что разрешение используемого спектрометра относительно невысокое, сдвиги $Q$-ветвей $\mathrm{N}_{2}\left(v_{1}\right)$ и $\mathrm{CO}_{2}\left(v^{(+)}\right)$, а также уширения обеих $Q$-ветвей $\mathrm{CO}_{2}$ явно видны. В свою очередь, спектры $\mathrm{H}_{2}$ как в окружении $\mathrm{CH}_{4}$, так и без него, существенных различий не имеют. Исходя из невязок в разностных спектрах $\mathrm{N}_{2}$ и $\mathrm{CO}_{2}$, следует, что неучет данных особенностей может привести к относительным погрешностям определения концентраций данных газов на уровне нескольких процентов, а также может замаскировать полосы иных компонентов ПГ.

\section{Заключение}

Проведенные исследования по изменению спектров КР $\mathrm{N}_{2}, \mathrm{CO}_{2}$ и $\mathrm{H}_{2}$ в окружении $\mathrm{CH}_{4}$ при фиксированном давлении $25 \mathrm{~atm}$ показали следующее. Частотный сдвиг $Q$-ветви колебательной полосы КР $v_{1} \mathrm{~N}_{2}$ уменьшается. $Q$-ветви ферми-дублета $\mathrm{CO}_{2} v_{1} / 2 v_{2}$ подвержены сужению. При этом частотный сдвиг $Q$-ветви высокочастотного компонента $v^{(+)}$уменьшается, а сдвиг низкочастотного компонента $v^{(-)}$остается без изменения. Помимо этого, в данных условиях уменьшается отношение их интенсивностей $I\left(v^{(+)}\right) / I\left(v^{(-)}\right)-$как пиковых, так и интегральных. Такие изменения спектров при определении компонентного состава ПГ посредством разложения его спектра КР по набору базисных спектров отдельных компонентов будут являться причиной увеличения погрешности измерений. В этой связи необходимо учитывать данные изменения либо путем внесения поправок в процедуру обработки спектров КР, либо посредством формирования базисных спектров в соответствующих условиях. В свою очередь, линии чисто вращательного спектра $\mathrm{H}_{2}$ не меняют своих спектральных характеристик в среде $\mathrm{CH}_{4}$ и могут быть использованы без дополнительных поправок.

Работа выполнена при поддержке РФФИ в рамках научного проекта № 16-32-60111 мол_а_дк.

\section{Список литературы}

[1] Petrov D.V., Matrosov I.I. // Appl. Spectrosc. 2016. V. 70. N 10. P. 1770. doi 10.1177/0003702816644611

[2] Петров Д.В., Матросов И.И., Тихомиров А.А. // ЖПС. 2015. T. 82. № 1. C. 124; Petrov D.V., Matrosov I.I., Tikhomirov A.A. // J. Appl. Spectrosc. 2015. V. 82. № 1. P. 120. doi 10.1007/s10812-015-0073-4

[3] Булдаков М.А., Королев Б.В., Корольков В.А., Матросов И.И., Петров Д.В., Тихомиров А.А. // Оптический журнал. 2013. Т. 80. № 7. С. 27; Buldakov M.A., Korolev B.V., Korolkov V.A., Matrosov I.I., Petrov D.V., Tikhomirov A.A. // J. Opt. Technol. 2013. V. 80. N 7. P. 426. doi 10.1364/JOT.80.000426

[4] Kiefer J., Seeger T., Steuer S., Schorsch S., Weikl M.C., Leipertz A. // Meas. Sci. Tech. 2008. V. 19. N 8. P. 085408. doi 10.1088/0957-0233/19/8/085408

[5] Eichmann S.C., Kiefer J., Benz J., Kempf T., Leipertz A., Seeger T. // Meas. Sci. Technol. 2014. V. 25. N 7. 075503-1. doi 10.1088/0957-0233/25/7/075503

[6] Buric M.P., Chen K.P., Falk J., Woodruff S.D. // Appl. Opt. 2009. V. 48. N 22. P. 4424. doi 10.1364/AO.48.004424

[7] Hippler M. // Anal. Chem. 2015. V. 87. N 15. P. 7803. doi 10.1021/acs.analchem.5b01462

[8] Sharma R., Poonacha S., Bekal A., Vartak S., Weling A., Tilak V., Mitra C. // Opt. Eng. 2016. V. 55. N 10. P. 104103. doi 10.1117/1.OE.55.10.104103

[9] Петров Д.В. // ЖПС. 2017. Т. 84. № 3. С. 399.

[10] Petrov D.V. // J. Raman Spectrosc. 2017. doi 10.1002/jrs.5141

[11] Seitz J.C., Pasteris J.D., Chou I.M. // Am. J. Sci. 1993. V. 293. N 4. P. 297. doi 10.2475/ajs.293.4.297

[12] Fabre D., Oksengorn B. // Appl. Spectrosc. 1992. V. 46. N 3. P. 468. doi 10.1366/0003702924125348

[13] Seitz J.C., Pasteris J.D., Chou I.-M. // Am. J. Sci. 1996. V. 296. N 6. P. 577. doi 10.2475/ajs.296.6.577

[14] Hansen S.B., Berg R.W., Stenby E.H. // Appl. Spectrosc. 2001. V. 55. N 6. P. 745. doi 10.1366/0003702011952442

[15] Howard-Lock H.E., Stoicheff B.P. // J. Mol. Spectrosc. 1971. V. 37. N 2. P. 321. doi 10.1016/0022-2852(71)90302-X

[16] Hacura A. // Phys. Lett. A 1997. V. 227. N 3-4. P. 237. doi 10.1016/S0375-9601(97)00010-8

[17] Petrov D.V., Matrosov I.I. // Proc. SPIE. 2016. V. 10003. P. 1003523. doi 10.1117/12.2249299

[18] May A.D., Degen N., Stryland J.C., Welsh H.L. // Can. J. Phys. 1961. V. 39. N 12. P. 1769. doi 10.1139/p61-200

[19] Petrov D.V., Matrosov I.I. // J. Raman Spectrosc. 2017. V. 48. N 3. P. 474. doi $10.1002 /$ jrs. 5062 